\title{
Human Stem Cell-Derived Retinal Pigment Epithelial Cells as a Model for Drug Screening and Pre-Clinical Assays Compared to ARPE-19 Cell Line
}

\author{
Carolina Reis Oliveira ${ }^{1, *}$, Mayara Rodrigues Brandão de Paiva ${ }^{2, *}$, Marcela Coelho Silva Ribeiro²,
} Gracielle Ferreira Andrade, Juliana Lott Carvalho ${ }^{4}$, Dawidson Assis Gomes ${ }^{1}$, Márcio Nehemy ${ }^{5}$, Ślvia Ligório Fialho ${ }^{6}$, Armando Silva-Cunha ${ }^{2}$, Alfredo Miranda de Góes ${ }^{1}$

${ }^{1}$ Department of Biochemistry and Immunology, Federal University of Minas Gerais, Belo Horizonte, Brazil

${ }^{2}$ Faculty of Pharmacy, Federal University of Minas Gerais, Belo Horizonte, Brazil

${ }^{3}$ SENAN, Centro de Desenvolvimento da Tecnologia Nuclear - CDTN/CNEN, Federal University of Minas Gerais, Belo Horizonte, Brazil

${ }^{4}$ Department of Genomic Sciences and Biotechnology, Catholic University of Brasilia, Brasilia, Brazil

${ }^{5}$ Department of Ophthalmology, Federal University of Minas Gerais, Belo Horizonte, Brazil

${ }^{6}$ Pharmaceutical Research and Development, Ezequiel Dias Foundation, Belo Horizonte, Brazil

Background and Objectives: Eye diseases have a high socioeconomic impact on society and may be one of the fields in which most stem cell-related scientific accomplishments have been achieved recently. In this context, human Pluripotent Stem Cell (hPSC) technology arises as an important tool to produce and study human Embryonic Stem cell derived-Retinal Pigmented Epithelial Cells (hES-RPE) for several applications, such as cell therapy, disease modeling, and drug screening. The use of this technology in pre-clinical phases attends to the overall population desire for animal-free product development. Here, we aimed to compare hES-RPE cells with ARPE-19, one of the most commonly used retinal pigmented epithelial immortalized cell lines.

Methods and Results: Functional, cellular and molecular data obtained suggest that hES-RPE cells more closely resembles native RPEs compared to ARPE-19. Furthermore, hES-RPE revealed an interesting robustness when cultured on human Bruch's membrane explants and after exposure to Cyclosporine (CSA), Sirolimus (SRL), Tacrolimus (TAC), Leflunomide (LEF) and Teriflunomide (TER). On these conditions, hES-RPE cells were able to survive at higher drug concentrations, while ARPE-19 cell line was more susceptible to cell death.

Conclusions: Therefore, hES-RPEs seem to have the ability to incur a broader range of RPE functions than ARPE-19 and should be more thoroughly explored for drug screening.

Keywords: ARPE-19, Human embryonic stem cell derived-retinal pigmented epithelial cells, In vitro toxicology, In vitro model

Received: May 30, 2020, Revised: October 7, 2020, Accepted: October 8, 2020, Published online: December 31, 2020

Correspondence to Mayara Rodrigues Brandão de Paiva

Faculty of Pharmacy, Federal University of Minas Gerais, 6627 Presidente Antônio Carlos Avenue, Pampulha, Belo Horizonte 31270-901, Brazil Tel: +55-31-34096949, Fax: +55-31-34996935, E-mail: mrbprodrigues@gmail.com

*These authors contributed equally to this work.

() This is an open-access article distributed under the terms of the Creative Commons Attribution Non-Commercial License (http://creativecommons.org/ licenses/by-nc/4.0/), which permits unrestricted non-commercial use, distribution, and reproduction in any medium, provided the original work is properly cited. Copyright (c) 2021 by the Korean Society for Stem Cell Research 


\section{Introduction}

Ocular toxicity tests are required to evaluate risks and ensure the safety of ophthalmic administration of drugs $(1,2)$. Several in vivo animal models have been used in this sense. However, species-related morphological and biochemical differences to the human eye compose major limitations of such models $(3,4)$. Furthermore, animal experiments have been extensively criticized in terms of cost, time and ethical issues (5). Thus, the search for alternative models for animal experiments has been encouraged for several fields, including ophthalmology. Cell culture models derived from human resources offer the advantage of constituting highly defined systems and may result in more reproducible data (6-9).

The retinal pigmented epithelium (RPE) is a monolayer of pigmented epithelial cells that reside between the neural retina and Bruch's membrane (BM). Even though RPE is not an intrinsic component of the visual signaling pathway, it is a highly metabolically active cell layer, which is vital to the health, survival, and function of the overlying photoreceptors $(10,11)$. Considering that RPE is critically important for normal function of the retina, intraocular drug or compound administration must be evaluated regarding possible toxicity against this cell layer (1, 12).

ARPE-19 was established and characterized in 1996 (6). Despite being considered a representative RPE cell line, these cells display poor transepithelial resistance values of $\sim 100 \Omega . \mathrm{m}^{2}$ and seem to lose RPE-specific genes when maintained in suboptimal culturing conditions (13). These limitations have encouraged the search for protocols for in vitro RPE generation from human pluripotent stem cells (hES-RPE) (14). hES-RPE closely resembles human fetal RPE and were capable of phagocytosis of fluorescently labeled rod outer segments. Therefore, hES-RPE have been investigated for cellular therapy, disease modeling and drug screening $(2,10,11,14-16)$.

Several diseases that cause ocular inflammation, including uveitis, scleritis, and orbital inflammatory disease result in impairment or loss of vision (17). The mainstay treatment is the use of corticosteroids, but the prolonged treatments and high doses of these drugs are associated with significant side effects (18). For this reason, corticosteroid-sparing agents like Cyclosporin (CSA) (19), Sirolimus (SRL) (20), Tacrolimus (TAC) (21), Leflunomide (LEF) (22) and its active metabolite teriflunomide (TER) have been investigated as alternatives to the use of corticosteroids.
While CSA, SRL, and TAC have already been applied for ocular diseases, there are few studies investigating LEF for this purpose (22-24). Nevertheless, the effect of these chemicals on RPE has not been verified in vitro, since the technology for producing functional hES-RPE is quite recent. Considering that, here, we aim to evaluate the differences between the RPE cell line ARPE-19 and hES-RPE cells, in order to underscore the importance of determining the best RPE source for preclinical toxicity drug testing. To do so, both RPE cell sources were characterized molecularly and functionally and used to assess the toxicity of the aforementioned drugs.

\section{Materials and Methods}

\section{Cell culture}

H1 (25) (National Institutes of Health-registered as WA01) were maintained in Matrigel (BD Biosciences, USA) using mTeSR (StemCell Technologies, USA) and subcultured using Dispase (BD Biosciences, USA). Differentiation protocol was performed by allowing $\mathrm{H} 1$ to overgrow until the hES colonies became multilayered. Culture media was then replaced with RPE differentiation medium (RPE medium), composed of knockout high glucose DMEM supplemented with $0.1 \mathrm{mg} / \mathrm{ml}$ Normocin (Invivogen, USA), 1\% nonessential amino acids solution, $2 \mathrm{mM}$ GlutaMAX-I (Invitrogen, USA), $0.1 \mathrm{mM}$ mercaptoethanol (Invitrogen, USA), 13\% Serum Replacement (Invitrogen, USA) and 5\% Fetal Bovine Serum (FBS) (Cripion Biotecnologia LTDA, BRA) (Fig. 1A).

ARPE-19 cell line, previously described as a human RPE cell line, was cultured in DMEM/F12 supplemented with 10\% Fetal Bovine Serum. The medium was changed every 2 days and cells were used between passages 9-19.

\section{Primary RPE isolation}

Primary RPE cells were isolated from fetal (18 22 weeks gestation) and adult (59 63 years old) eyes (Advanced Biosciences Resources, Inc, USA). Cells were obtained following RPE layer digestion using collagenase IV (Gibco, USA) at $0.8 \mathrm{mg} / \mathrm{ml}$ (fetal eyes) or $0.4 \mathrm{mg} / \mathrm{ml}$ (adult eyes). RPE cells were cultured using RPE medium and tissue culture flasks covered with the extracellular matrix produced by bovine corneal endothelial cells (26). After reaching confluence, cultures were expanded using $0.25 \%$ trypsin-EDTA.

\section{Quantitative PCR}

Cells were harvested from fetal and adult RPE (fRPE 


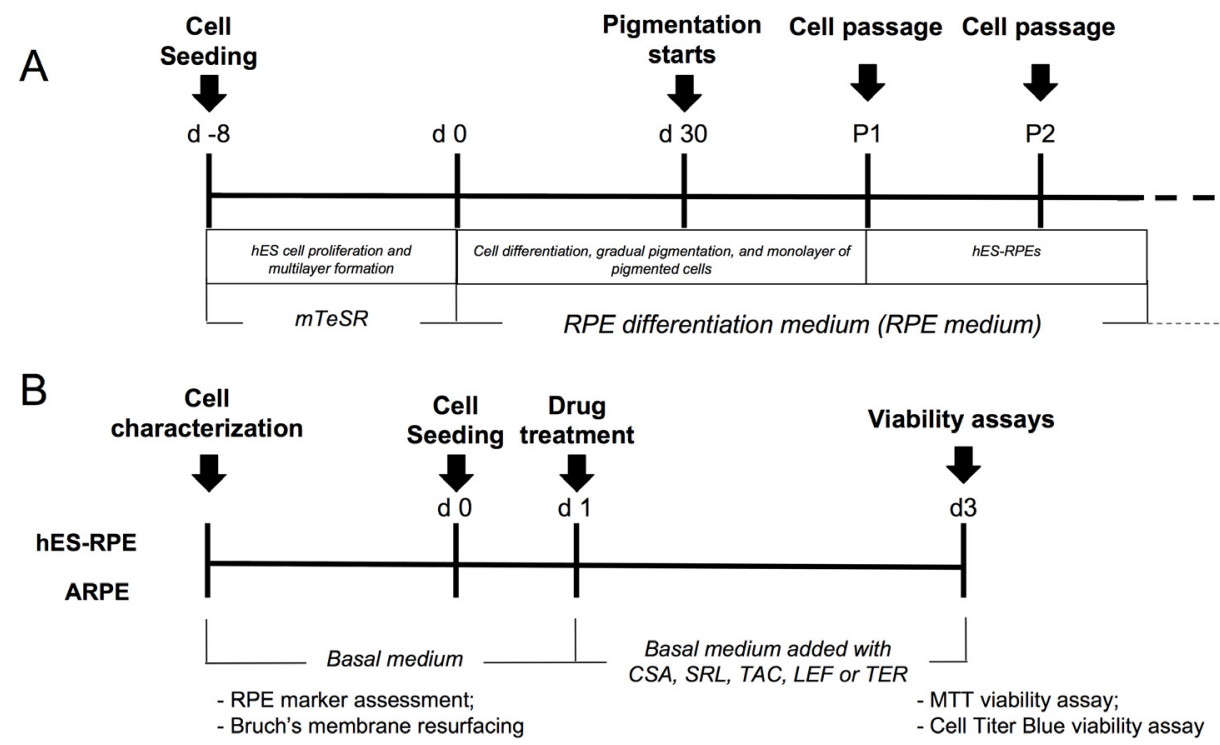

Fig. 1. hES-RPE differentiation and experimental design. (A) hES-RPE were differentiated by proliferation and multilayer formation of hES cells and removal of bFGF of the medium. (B) hES-RPE and ARPE-19 were first characterized according to mRNA expression of RPE markers, then according to Bruch's membrane resurface ability. Finally, hES-RPE and ARPE-19 were used for cytotoxicity assays.

and ARPE, respectively), ARPE-19 and hES-RPE cultures for mRNA characterization (Fig. 1B). fRPE were collected at Passage 1, day 4 for experiments. ARPE were dissected from a donor eye (age 59 63) and directly processed for RNA isolation using Trizol. Total RNA samples were treated with DNase (Promega, USA) and quantified by spectrophotometry. cDNA was obtained using the RevertAid $^{\mathrm{TM}}$ H Minus M-MuLV RT (Fermentas, USA). Next, PCR amplification for pluripotent stem cell markers OCT-4 and NANOG, and RPE markers RPE-65, bestrophin, CRLBP, MITF, PEDF, and ZO-1, was performed using the TaqMan Gene Expression Assay kit (Applied Byosystems, USA). The relative level of gene expression was determined and normalized to 18s rRNA. Each sample was run in technical triplicates and biological duplicates. Comparisons were made considering samples processed at the same time.

\section{Western blotting}

The protein expression of MITF and BEST was also evaluated using Western Blotting. Cell lysates were prepared from RPE-hESC in passages 1 to 3 . The cells were lysed on ice using cold lysis buffer $(20 \mathrm{mM}$ Tris, $1 \mathrm{mM}$ EDTA, $150 \mathrm{mM} \mathrm{NaCl}, 1 \%$ Triton X-100, 10\% glycerol) containing protease inhibitor (Protease Inhibitor cocktail II, Calbiochem). Then, samples were vortexed for $15 \mathrm{sec}-$ onds and incubated on ice for $5 \mathrm{~min}$. After repeating this step two more times, the lysate was centrifuged for $15 \mathrm{~min}$ at $13500 \mathrm{~g}$ at $4^{\circ} \mathrm{C}$. Protein concentration was measured by Bradford assay (BioRad). Cell lysates $(30 \mu \mathrm{g})$ were mixed with Laemmli buffer, added with $\beta$-mercaptoethanol and boiled for $5 \mathrm{~min}$. Proteins were separated using 12\%
SDS-PAGE polyacrylamide gel and then transferred to a nitrocellulose membrane for $2 \mathrm{~h}$ at $75 \mathrm{~V}$ in transfer buffer (25 mM Tris, $192 \mathrm{mM}$ glycine, 20\% methanol, pH 8.3). The membrane was washed with PBS-T (Tween-20 0.05\%) and blocked with 5\% BSA in PBS-T for $1 \mathrm{~h}$. The membranes were incubated with mouse anti-RPE-65 1:500 (Pierce) and mouse anti-CRLBP 1:750 (Abcam) overnight at $4^{\circ} \mathrm{C}$. After 3 washes of $15 \mathrm{~min}$ in PBS-T, the membranes were incubated with secondary antibody anti-mouse IgG diluted $1: 10,000$. Chemiluminescence was developed using the ECL Plus kit (Amersham Biosciences) and the signal scanned and collected using a Typhoon imager (Amersham Biosciences).

\section{Immunofluorescence}

RPE markers were also analyzed in hESC-RPE using immunofluorescence. For this, passage 2 RPE cells were fixed with $4 \%$ paraformaldehyde for $15 \mathrm{~min}$, permeabilized with $0.1 \%$ Triton X-100, and blocked with a solution containing 2\% goat serum (Normal Goat Serum NGS) $0.5 \%$ BSA, and $0.1 \%$ Triton X-100 diluted in PBS for 1 hour. Primary antibodies were diluted in 2\% NGS / PBS-T $0.3 \%$ and incubated at $4{ }^{\circ} \mathrm{C}$ overnight, using the following dilutions: $1: 50$ of the rabbit polyclonal transcription factor associated with microphthalmia, MITF (Abcam); 1:250 of mouse monoclonal bestrofin membrane protein, BEST (Pierce). Goat anti-mouse IgG FITC ( $1: 32$; Sigma), and goat anti-rabbit IgG rhodamine (Jackson ImmunoResearch Laboratories) were diluted in blocking solution and incubated with samples for 1 hour at room temperature. Nuclear staining was executed using $0.2 \mu \mathrm{g} / \mathrm{ml}$ DAPI (Sigma) diluted in PBS. The slides were 
assembled using Vectashield (Vector Laboratories). The images were obtained using a confocal microscope (Confocal Zeiss 5 LIVE).

\section{Melanin content analysis}

Melanin content is one of the criteria used to select and characterize hESC-RPE batches used for cellular therapy (27-29). In order to quantify the intracellular melanin content of hESC-RPE, passage 2 cells were harvested on days 8,12 and 16 after passage. The cells were centrifuged at $160 \mathrm{~g}$ for $5 \mathrm{~min}$ at room temperature and counted. Pellets were resuspended in $1 \mathrm{M} \mathrm{NaOH}$ and heated at $80^{\circ} \mathrm{C}$ for $10 \mathrm{~min}$, vortexed, and the absorbance measured at $475 \mathrm{~nm}$ against a standard synthetic melanin curve (Sigma) ranging from 5 to $180 \mu \mathrm{g} / \mathrm{ml}$. Samples were analyzed in triplicates and the data normalized to the total number of cells.

\section{VEGF synthesis}

hESC-RPE were plated at $6 \times 10^{4}$ cells $/ \mathrm{cm}^{2}$ on Transwell plates (Corning) prepared with Matrigel or BM substrates (Sigma). The cell culture supernatants on the apical and basal sides (the compartment above and below the Transwell, respectively), were collected 48 hours after plating and the samples assayed, in duplicates, with the DuoSet ELISA Human VEGF R\&D Systems kit.

\section{Explant assay - Bruch's membrane organ culture}

The ability of ARPE-19 and hES-RPE cells to resurface aged human BM was assessed since this is the area where $\mathrm{RPE}$ is first affected in diseases like AMD, and also as an additional evidence of the resemblance of ARPE-19 and hES-RPE to the native RPE. In this assay, cellular attachment and survival were analyzed by scanning electron microscopy (SEM).

In order to prepare the ex vivo culture experiment,

B
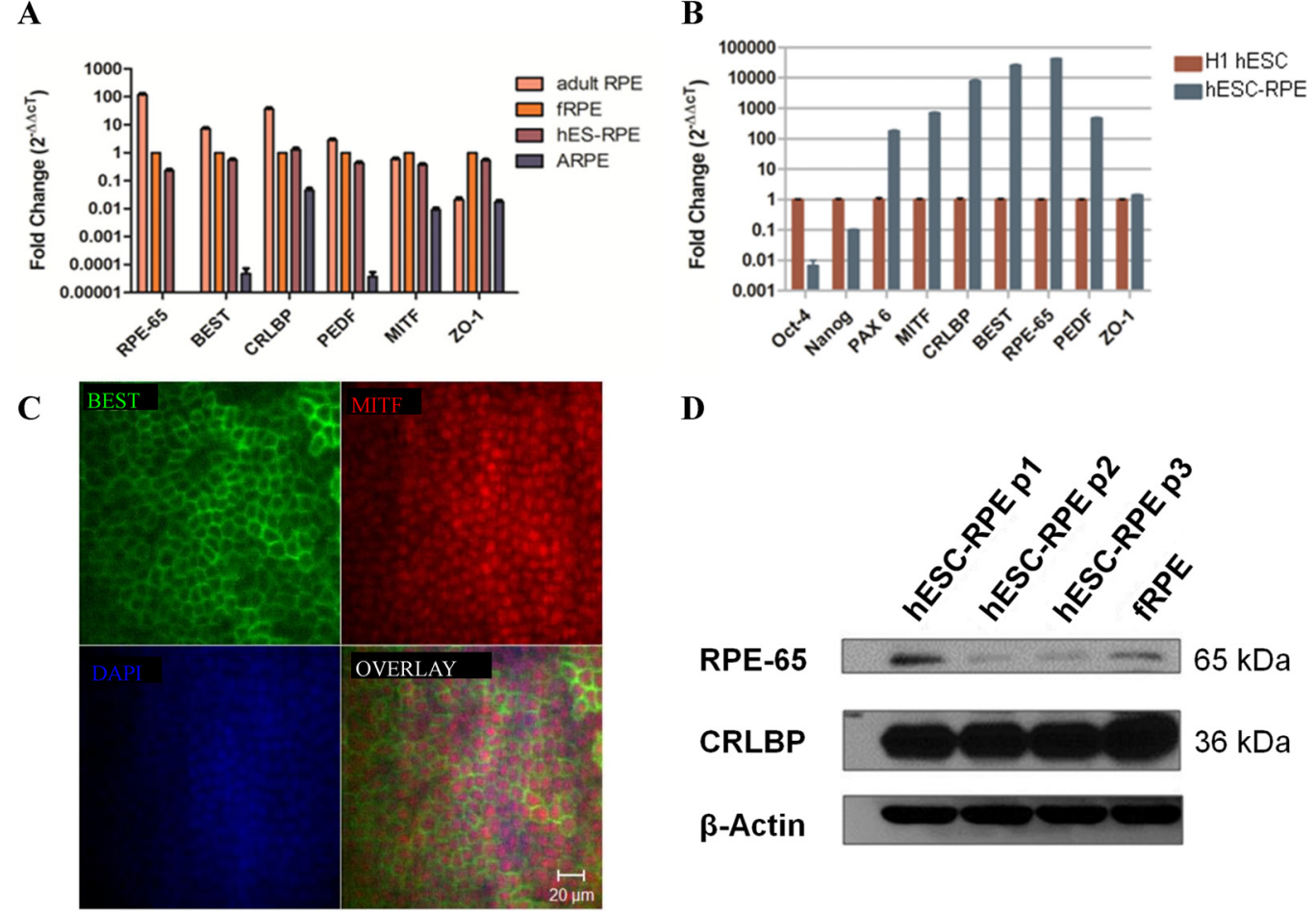

Fig. 2. mRNA and protein expression of RPE markers. (A) The mRNA expression of RPE-65, BEST, CRLBP, MITF, PEDF, and ZO-1 was evaluated by quantitative real-time polymerase chain reaction. mRNAs were quantified from hES-RPE, ARPE-19, fetal RPE, and adult RPE normalized to the geometric mean of a housekeeping gene (18s rRNA). (B) The mRNA expression of pluripotency (OCT4 and NANOG), neuroectoderm (PAX6) and RPE markers (MITF, CRLBP, BEST, RPE-65, PEDF and ZO-1) was also compared between undifferentiated hESCs and hESC-RPE. As expected, compared to undifferentiated $\mathrm{H} 1$, differentiated cells at passage 2 presented lower expression of pluripotency genes, and higher expression of neuroectoderm and RPE differentiation markers. (C) Protein expression of BEST and MITF was evaluated in p2 hESC-RPE cells by immunofluorescence. As expected, BEST presented membrane localization, and MITF, nuclear localization. (D) Western Blotting analysis of RPE-65 and CRLBP expression by p1, p2 and p3 hESC-RPE cells. Similar to fetal RPE, hESC-RPE cells from all the analysed passages presented detectable expression of both RPE markers. Bars represent standard error of the mean. Abbreviations: hES-RPE, RPE derived from human embryonic stem cells; fRPE, fetal RPE. 
adult donor eyes (age 59 63) were received from the eye bank of the city of Belo Horizonte, MG - Brazil (Ethical Committee approval no. ETIC 33734514.7.0000.5149). Acceptance criteria followed previous studies (30, 31). Six-millimeter-diameter corneal trephines (Bausch and Lomb, USA) was used to create macula-centered BM explants which were debrided. Explants were seeded with 3,164 cells $/ \mathrm{mm}^{2}$, shown to yield a monolayer of cells with $24 \mathrm{~h}$ after seeding (30). Explants were harvested at day 7, fixed in paraformaldehyde-glutaraldehyde Karnovsky's Fixative solution and processed for SEM.

Scanning electron microscopy: Explants were fixed as mentioned and washed 3 times with PBS and incubated with $1 \%$ osmium tetroxide (Electron Microscopy Sciences, USA) in PBS at $4^{\circ} \mathrm{C}$ in the dark for $1 \mathrm{~h}$. After, samples were washed with PBS and incubated in $1 \%$ tannic acid in water. Explants were subsequently washed with PSB and incubated in $1 \%$ osmium tetroxide in water at $4^{\circ} \mathrm{C}$ in the dark for $1 \mathrm{~h}$. Then, samples were washed with PBS and dehydrated through stepwise incubation in a series of graded ethanol baths at $35 \%, 50 \%, 70 \%, 85 \%, 95 \%$ and $100 \%$ concentration. Samples were submitted to critical point drying using $\mathrm{CO}_{2}$ (Leica EM CPD030, USA). Finally, explants were coated with gold (BALTEC MED020 Coating System, LIE) and analyzed in the Microscopy Center of Biological Sciences Institute, Federal University of Minas Gerais, Brazil (DSM950- Zeiss, GER).

\section{Drug toxicity testing}

hES-RPE cells and ARPE-19 were seeded on 96-well plates at $1 \times 10^{4}$ cells/well. After $24 \mathrm{~h}$, the medium was changed and CSA, SRL, TAC, LEF, and TER were added at increasing doses of $31.6 \mu \mathrm{M}\left(10^{1.5}\right), 56 \mu \mathrm{M}\left(10^{1.75}\right), 100$ $\mu \mathrm{M}\left(10^{2}\right), 177.8 \mu \mathrm{M}\left(10^{2.25}\right)$ and $316 \mu \mathrm{M}\left(10^{2.5}\right)$. Cells were incubated with respective drugs for $72 \mathrm{~h}$ and had their viability assessed by the 3-(4,5-dimethylthiazol-2-yl)2,5-diphenyl tetrazolium bromide (MTT) assay (32) or CellTiter Blue, following manufacturer's instructions. Data were obtained from three independent experiments. The IC50 (concentration of the drug in which cell viability decreased by $50 \%$ ) was determined for each drug incubated with each cell.

\section{Statistical analysis}

Statistical analysis was performed by analysis of variance (ANOVA) followed by Bonferroni's post-test using Graph Pad Prism 5.0. Values were represented as mean standard deviation. Differences were considered significant if $\mathrm{p}<0.05$.

\section{Results}

\section{Analysis of RPE marker expression in hES-RPE and ARPE-19}

hES-RPE and ARPE-19 are considered relevant experimental models of the native RPE, for in vitro investigations. In order to assess the similarity of hESRPE and ARPE-19 relative to ARPE and in situ ARPE, these cells were obtained and characterized morphologically, molecularly and functionally (Fig. 1B, Supplementary Fig. S1). mRNA expression of proteins involved in visual cycle (CRLBP and RPE-65), RPE-specific transcription factors (MITF), membrane-associated proteins (BEST), secreted factors (PEDF) and tight junction proteins (ZO-1) was performed. Obtained data showed that hES-RPE mRNA levels were similar to fRPE and ARPE (Fig. 2A). On the other hand, ARPE-19 revealed lower expression levels of all RPE markers compared to other groups, with ZO-1 being the only exception, presenting statistically similar expression between ARPE and ARPE-19. Despite constituting an important gene for RPE function, due to its contribution to tight junction formation and integrity of the blood-retina barrier, ZO-1 is not a specific RPE marker. Compared to undifferentiated pluripotent stem cells, hESC-RPE presented lower expression of pluripotency markers, as well as higher expression of RPE markers (Fig. 2B).

The RPE differentiation was also investigated using protein markers. Immunofluorescence analysis confirmed that hESC-RPE cells expressed BEST, MITF, with membrane and nuclear localization, respectively (Fig. 2C).

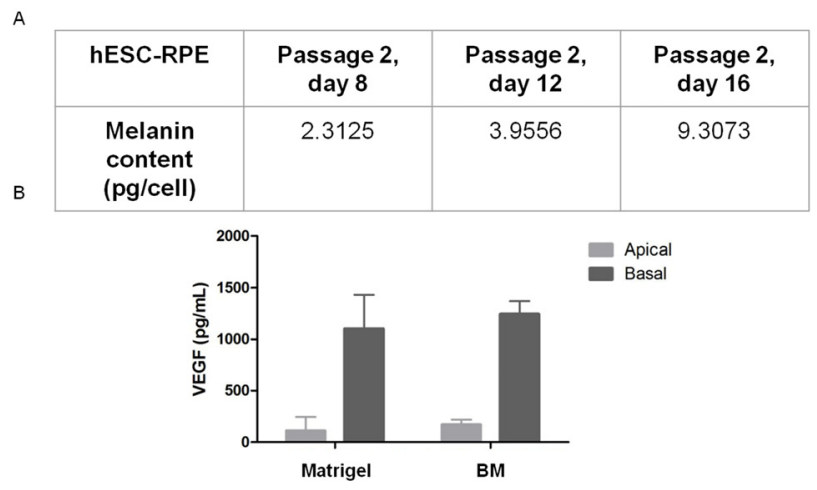

Fig. 3. Functional analysis of hESC-RPE cells. (A) Melanin content of passage 2 hESC-RPE cells in different times of culture. (B) Polarized VEGF synthesis of hESC-RPE cells. As depicted, hESC-RPE cells preferentially secrete VEGF in the basal side. Bars represent standard error of the mean. Abbreviations: hES-RPE, RPE derived from human embryonic stem cells; BM, human Bruch's membrane. 
Finally, the hESC-RPE differentiation was confirmed using western blotting analysis of RPE-65 and CRLBP, which were expressed by hESC-RPE in different passages, similar to fRPE cells (Fig. 2D).

Pigment production is also a determining factor in the maturation stage of cells, this being one of the criteria used to determine the degree of differentiation that cells should be used for therapy. Therefore, the functionality of hESC-RPE was analyzed according to the melanin content of cells in different time-points of differentiation (Fig. 3A), and revealed that the differentiated cells accumulated intracellular melanin over time. The verified melanin content surpassed the minimal hESC-RPE melanin content for clinical application (27-29).
RPE cells in situ are known to secrete growth factors, such as VEGF, in a polarized manner (10). Similarly, RPE monolayers cultured in vitro show preferential secretion of VEGF in the basal side (33). In order to verify the functional character of the EPR-hESC cells, they were seeded in transwells and the culture medium was collected from both upper and lower reservoirs, as previously described (34). The hESC-RPE secreted VEGF preferentially in the basal side, with a basal/apical ratio ranging from 7.4 to 9.9 (Fig. 3B). The production of VEGF on both the apical and basal side was similar for cells cultured on both types of MEC substrates of Matrigel and also BM. The resurfacing experiments of $\mathrm{hBM}$ are further described below.
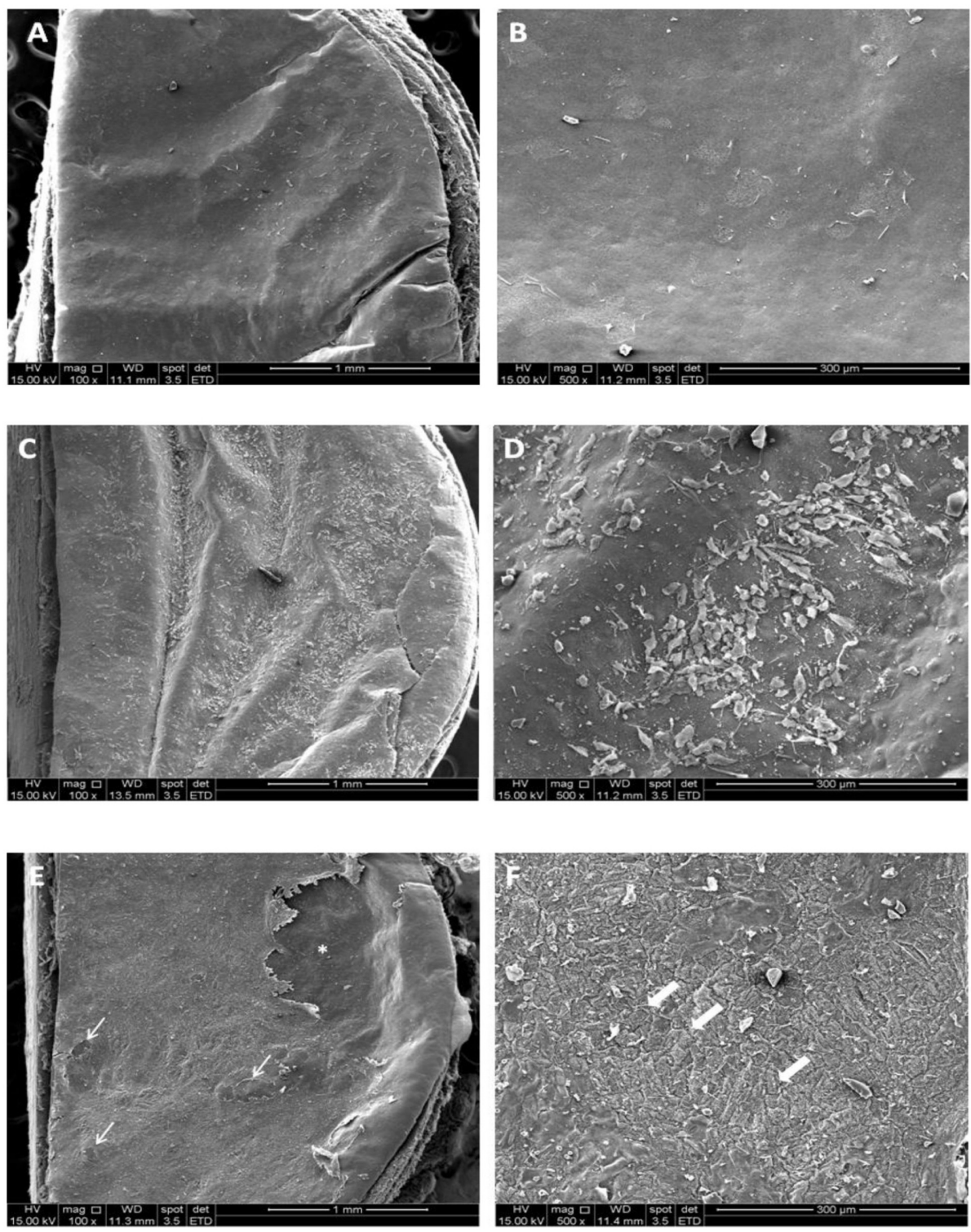

Fig. 4. Analysis of RPE cells' ability to resurface aged human Bruch's membrane (BM) explants. (A, B) SEM micrography of the superficial structure of the BM's illustrating exposition of the basement membrane layer. (C, D) ARPE-19 cells seeded and cultured on BM's were not able to proliferate and the explant was only partially resurfaced; (E, F) hESRPE cells seeded and cultured on $B M^{\prime}$ s were able to survive, proliferate and virtually resurface the whole explant, with small flaws (narrow arrows). hES-RPE formed a cell layer with a morphology that resembles the hexagonal character of native fetal RPE (wide arrows). *Represents an artifact occurred during manipulation of the sample. 


\section{MTT Tetrazolium Assay}

A

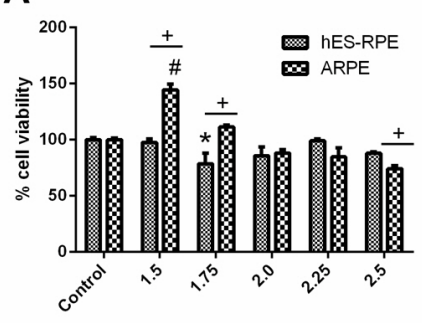

Cyclosporin $\left(10^{\times} \mu \mathrm{M}\right)$

D
B

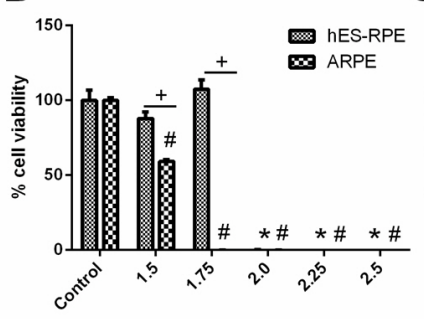

Sirolimus $\left(10^{\mathrm{x}} \mu \mathrm{M}\right)$
C

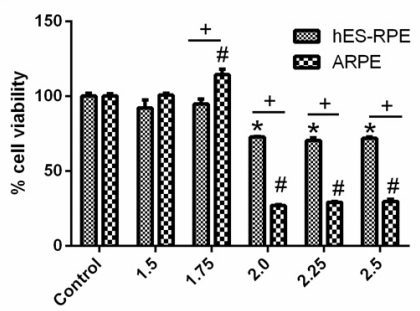

Tacrolimus $\left(10^{\times} \mu \mathrm{M}\right)$

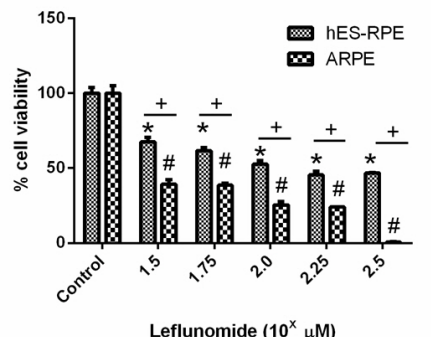

E

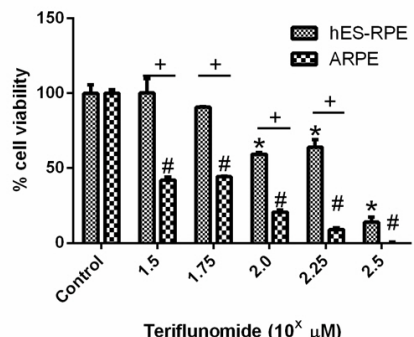

\section{Resazurin Reduction Assay}

A

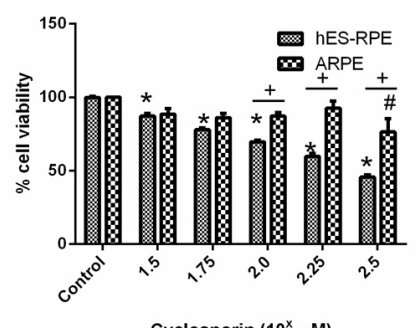

B

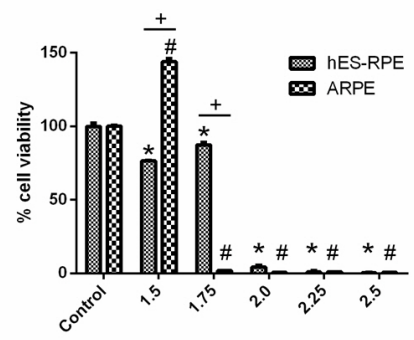

C

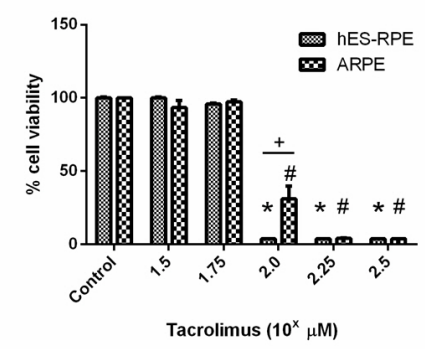

D

E

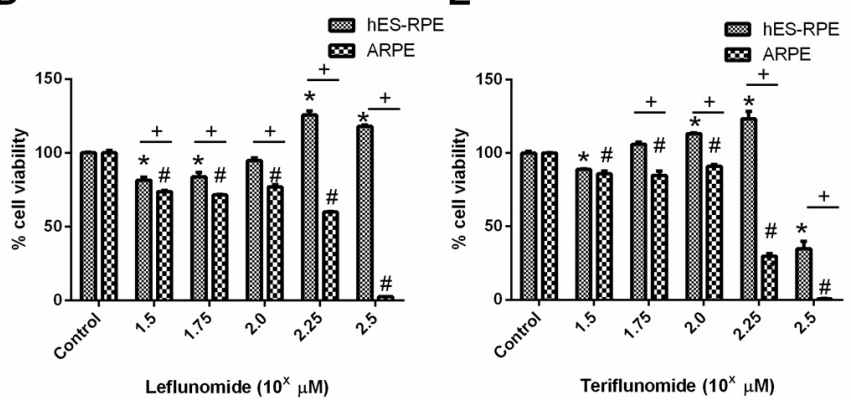

Fig. 5. Drug cytotoxicity analysis of sirolimus, leflunomide, and teriflunomide evaluated by MTT Tetrazolium Assay (I) and Resazurin Reduction Assay (CellTiter-Blue ${ }^{\mathbb{R}}$ Cell Viability Assay) (II). Cyclosporin was not cytotoxic for both cell types in the analyzed doses, however, showed a reduction of cell viability greater than $50 \%$ for hES-RPE at the dose of $100 \mu \mathrm{M}$. Sirolimus (IB and IIB) showed higher cytotoxicity than leflunomide and its active metabolite, teriflunomide. TAC treatment (IC and IIC) showed cytotoxic for ARPE-19 at dose evaluated $100 \mu \mathrm{M}\left(10^{2}\right)$ while it was cytotoxic for hES-ARPE at dose evaluated $176 \mu \mathrm{M}\left(10^{2.25}\right)$. Leflunomide treatment (ID and IID) was cytotoxic for ARPE-19 at the highest dose evaluated $\left(178 \mu \mathrm{M}, 10^{2.25}\right)$ while it was not cytotoxic for hES-ARPE cells at this concentration. Teriflunomide treatment (IE and IIE), however, showed cytotoxicity for both cell types with the dose of $178 \mu \mathrm{M}\left(10^{2.25}\right)$, and at $100 \mu \mathrm{M}\left(10^{2}\right) \mathrm{ARPE}-19$ cells were also susceptible, but not hES-RPE cells. *significant difference comparing hES-RPE control and hES-RPE treatments; \#significant difference comparing ARPE-19 control and ARPE-19 treatments; +significant difference comparing hES-RPE and ARPE-19 at same concentration. 
Aged BM resurfacing by hES-RPE cells and ARPE-19

The capacity of hES-RPE and ARPE-19 to resurface the BM surface was assessed in order to investigate their ability to attach and survive on the area where RPE cells are critical for the maintenance of vision, the macula. To do so, hES-RPE and ARPE-19 were seeded on paired donor aged BM after a debridement protocol (Fig. 4A and 4B). SEM analysis revealed that ARPE-19 cells failed to attach and survive in this biologically relevant substrate, once only a few cells could be seen spread on BM (Fig. 4C and 4D). On the other hand, not only hES-RPE cells were able to completely resurface BM but also revealed a morphological resemblance to the native RPE (Fig. 4E and 4F).

\section{Viability assessment of hES-RPE and ARPE-19 treated with different drugs}

Following the introduction of in vitro assays, which aroused as alternative methods for in vivo toxicity assessment, several groups moved to in vitro models to test drug toxicity and efficiency (4). Since our previous experiments have highlighted phenotypic and functional differences between hES-RPE and ARPE-19, we proceeded with the analysis of another possible difference between those cells - their sensibility to specific drugs. Our data showed that CSA, SRL, TAC, LEF, and TER significantly decreased cell viability in vitro in a dose-dependent manner (Fig. 5 I e II). Interestingly, for most of the tested drugs, ARPE19 cells viability was accentuated reduced compared to hES-RPE cells, particularly at higher drug dosages. Indeed, according to MTT cell viability assay, TER treatment (Fig. 5 IE and IIE) was significantly toxic to ARPE19 at $>31.6 \mu \mathrm{M}(\mathrm{p}<0.0001)$, while to hES-RPE cells, significantly decreased cell viability at $>100 \mu \mathrm{M}(\mathrm{p}<$ 0.0001) (IC $\mathrm{I}_{50}$ values were $133.2 \mu \mathrm{g} \cdot \mathrm{ml}^{-1}$ for hES-RPE and $103.9 \mu$ g.ml ${ }^{-1}$ for ARPE-19). LEF treatment (Fig. 5 ID and IID) results also confirmed this evidence and showed that, considering each tested concentration of this drug, ARPE-19 showed less cell viability level than hES-RPE cells, for MTT and CellTiter-Blue ${ }^{\mathbb{R}}$ cell viability assays. LEF significantly reduced cell viability in both assays and for both cells at concentrations $>31.6 \mu \mathrm{M}(\mathrm{p}<0.0001)$, and showed $\mathrm{IC}_{50}>316 \mu \mathrm{M}$ for hES-RPE cells, while for ARPE-19 the IC 50 value was $166.70 \mu \mathrm{M}$.

Regarding SRL (Fig. 5 IB and IIB), the most toxic drug among those evaluated, we observed different cytotoxic effects on retinal cells at the lowest concentration of this chemical, according to the MTT cell viability assay. However, at $56 \mu \mathrm{M}, \mathrm{hES}-\mathrm{RPE}$ cells were more resistant to SRL compared to ARPE-19 cells, for both assays. SRL was significantly toxic for ARPE-19 cells at concentrations >56 $\mu \mathrm{M}(\mathrm{p}<0.0001)$, and $>100 \mu \mathrm{M}$ for hES-RPE $(\mathrm{p}<$ 0.0001 ) ( $\mathrm{IC}_{50}$ value of $75.1 \mu \mathrm{g} \cdot \mathrm{ml}^{-1}$ for hES-RPE while the $\mathrm{IC}_{50}$ value for ARPE-19 was lower, $48.7 \mu \mathrm{g} \cdot \mathrm{ml}^{-1}$ ).

On the other hand, the results suggested that hES-RPE cells were more sensitive to CSA and TAC treatments, considering resorufin assay. TAC treatment (Fig. 5 IC and IIC) significantly reduced cell viability on both cell lines at concentrations $>100 \mu \mathrm{M}(\mathrm{p}<0.0001)$, showing $\mathrm{IC}_{50}=$ $127.74 \mu \mathrm{M}$ for ARPE-19 and $\mathrm{IC}_{50}=117.03 \mu \mathrm{M}$ for hESRPE. The CSA treatment (Fig. 5 IA and IIA) was the least toxic drug tested and the viability of the tested cells was statistically different at concentrations $>10 \mu \mathrm{M}$ ( $\mathrm{p}=$ 0.0093) on CellTiter-Blue ${ }^{\circledR}$ assay, showing $\mathrm{IC}_{50}>316 \mu \mathrm{M}$ for ARPE-19 and $260.4 \mu \mathrm{M}$ for hES-RPE. Even with these differences, MTT assay demonstrated at higher concentrations of these drugs that ARPE-19 cells were more sensitive to all drugs tested. Therefore, it can be observed that, overall, ARPE-19 revealed higher sensibility to toxicological assault than hES-RPE.

\section{Discussion}

RPE cells constitute a highly desirable source of cells for therapy in $\mathrm{AMD}(14,15)$, but may also be used as an animal-free option for ocular drug discovery, toxicity screening and therapy $(4,35)$. hES-RPE and ARPE-19 are considered two suitable options for in vitro RPE modeling (36). However, properties of the ARPE-19 line seem to change according to the way cells are maintained. In different laboratories, this line is reported to exhibit different cell morphologies, ranging from those that resemble the native RPE to morphologies that are barely compatible with an epithelial phenotype (37). Here, ARPE-19 were correctly maintained and used at the low passage to prevent loss of RPE-markers and behavior. In order to verify the molecular profile of each cell line, we have assessed the expression of RPE-specific markers. The RPE phenotype was also assessed morphologically, molecularly and functionally, by investigating hES-RPE and ARPE-19 VEGF production, melanin synthesis, as well as cellular behavior when seeded on human BM. Finally, susceptibility to chemical treatments was investigated, in order to assess the suitability of each cell model for in vitro toxicity research (Fig. 1B).

The level of mRNA expression of RPE-markers was assessed by Real-time PCR and compared to primary human RPE cells from both fetal and adult tissues since it is known that RPE goes through significant maturation during life. Obtained results show that hES-RPE cells differentiated in our hands present an expression status highly 
similar to fetal RPE, but also intermediate compared to adult RPE, allowing us to classify such cells as "young RPE”. In an opposite direction, ARPE-19 mRNA profile was far different from both primary RPE cells, confirming that this cell line has lost important RPE markers, with the exception of ZO-1, under aforementioned conditions of cellular maintenance and expansion. As expected, hESC-RPE presented a lower expression of pluripotency genes, compared to undifferentiated counterparts.

Even though mRNA expression may be an important indication of cellular phenotype, protein and functional assays are important for phenotype validation. Therefore, the protein expression of MITF and BEST were assessed using immunofluorescence, and the expression of RPE-65 and CRLBP were assessed using western blotting. Both analysis confirmed the mRNA expression analysis, further supporting the notion that hESC-RPE were differentiated and presented an RPE phenotype.

The functionality of both hES-RPE and ARPE-19 was investigated according to the capacity of polarized VEGF synthesis and melanin content. Furthermore, cellular behavior of both cell samples was assessed when they were seeded on a surface that mimics the area where RPE cells are originally found in the ocular globe. Due to the fact that the changes in $\mathrm{BM}$ engendered by aging and $\mathrm{AMD}$ are complex and may not be fully reversible $(38,39)$, any source of RPE able to regenerate this area is desired. In this sense, not only BM adhesion may add a functional evidence to support their similarity compared to functional native RPE, but also reveals a possible suitability of those cells for AMD cellular therapy. SEM analysis showed that hES-RPE were able to resurface aged BM, in stark contrast to ARPE-19, which failed to attach, survive and proliferate on this surface.

Finally, the use of stem cell-derived RPE for drug screening constitutes a powerful approach for the development of new agents. Indeed, previous studies have been shown that the safety and efficacy can be enhanced by the use of relevant human cellular models, at the same time that the use of animals for such purpose can be reduced $(27,40) \mathrm{CsA}, \mathrm{SRL}, \mathrm{TAC}$, and LEF are drugs with known immunosuppressive properties, fully explored as corticosteroid-sparing agents for the treatment of many inflammation processes, including ocular diseases. However, these drugs had their use limited by the poor water solubility and severe side effects. Considering their intraocular administration, it becomes crucial to analyze their cytotoxicity, for instance, by evaluating each chemical in contact with the RPE cells. According to MTT assay results, ARPE-19 is more sensitive to the toxicity effects of tested drugs compared to hES-RPE. This behavior was observed in both MTT and CellTiter-Blue ${ }^{\circledR}$ cell viability assays for the majority of the tested chemicals. Despite differences, both MTT and CellTiter Blue assays indicate a more "robust" phenotype of hES-RPE cells compared to ARPE-19.

In vivo, one of the major functions of the RPE layer is the composition of blood-retina-barrier, which regulates the movement of solutes between the fenestrated capillaries of the choroid and the photoreceptor layer of the retina. Several membrane-associated transport proteins, such as multidrug resistance-associated proteins (MRPs), have been characterized at the blood-retinal-barrier and play an important role in regulating tissue bioavailability of several pharmacologic agents (41). Furthermore, has been described that the recorded levels of MRPs are significantly higher in hES-RPE compared with ARPE-19, mainly in most mature cobblestone hES-RPE. Considering that these ATP-dependent efflux transporters have a major role in drug delivery in human RPE, we can suggest that the presence of these proteins could be related to the observed results.

In summary, we have highlighted how hES-RPE and ARPE-19 cells show different profiles in vitro, in a way that the former can closely resemble native RPE while the latter fails in this aspect. It is important to use a cell source able to faithfully mimic the behavior of a healthy human cell type in order to predict important effects during preclinical research. In addition, the phenotype analysis compared to native cell types also reinforces the choice of the best source of cells for transplantation, in order to achieve better success rates in clinical phases. The generated hES-RPE seems to present the desirable features for these purposes and should, therefore, be fully explored, substituting any cell lineage that may be less representative, as well as the increasingly condemned animal-based models.

\section{Acknowledgments}

The authors acknowledge the support from the Midwest Eyebank (includes eye banks in Illinois, Michigan, and New Jersey-USA) and for the financial support from the Conselho Nacional de Desenvolvimento Científico e Tecnológico (CNPq/Brazil), Fundação de Amparo à Pesquisa de Minas Gerais (FAPEMIG/Brazil), Coordenação de Aperfeiçoamento de Pessoal de Nível Superior (CAPES) and Pró-reitoria de Pesquisa da Universidade Federal de Minas Gerais. 


\section{Potential Conflict of Interest}

The authors have no conflicting financial interest.

\section{Supplementary Materials}

Supplementary data including one figure can be found with this article online at https://doi.org/10.15283/ijsc20094

\section{References}

1. Wilson SL, Ahearne M, Hopkinson A. An overview of current techniques for ocular toxicity testing. Toxicology 2015; 327:32-46

2. Aberdam E, Petit I, Sangari L, Aberdam D. Induced pluripotent stem cell-derived limbal epithelial cells (LiPSC) as a cellular alternative for in vitro ocular toxicity testing. PLoS One 2017;12:e0179913

3. del Amo EM, Vellonen KS, Kidron H, Urtti A. Intravitreal clearance and volume of distribution of compounds in rabbits: In silico prediction and pharmacokinetic simulations for drug development. Eur J Pharm Biopharm 2015;95(Pt B):215-226

4. Barar J, Asadi M, Mortazavi-Tabatabaei SA, Omidi Y. Ocular drug delivery; impact of In vitro cell culture models. J Ophthalmic Vis Res 2009;4:238-252

5. Combes $\mathrm{RD}$, Shah $\mathrm{AB}$. The use of in vivo, ex vivo, in vitro, computational models and volunteer studies in vision research and therapy, and their contribution to the Three Rs. Altern Lab Anim 2016;44:187-238

6. Dunn KC, Aotaki-Keen AE, Putkey FR, Hjelmeland LM. ARPE-19, a human retinal pigment epithelial cell line with differentiated properties. Exp Eye Res 1996;62:155-169

7. Hosoya K, Tomi M, Ohtsuki S, Takanaga H, Ueda M, Yanai N, Obinata $M$, Terasaki T. Conditionally immortalized retinal capillary endothelial cell lines (TR-iBRB) expressing differentiated endothelial cell functions derived from a transgenic rat. Exp Eye Res 2001;72:163-172

8. Pfeffer BA, Philp NJ. Cell culture of retinal pigment epithelium: special issue. Exp Eye Res 2014;126:1-4

9. Hartnett ME, Lappas A, Darland D, McColm JR, Lovejoy S, D'Amore PA. Retinal pigment epithelium and endothelial cell interaction causes retinal pigment epithelial barrier dysfunction via a soluble VEGF-dependent mechanism. Exp Eye Res 2003;77:593-599

10. Strauss O. The retinal pigment epithelium in visual function. Physiol Rev 2005;85:845-881

11. Hansson ML, Albert S, González Somermeyer L, Peco R, Mejía-Ramírez E, Montserrat N, Izpisua Belmonte JC. Efficient delivery and functional expression of transfected modified mRNA in human embryonic stem cell-derived retinal pigmented epithelial cells. J Biol Chem 2015;290: 5661-5672

12. Lakkaraju A, Umapathy A, Tan LX, Daniele L, Philp NJ, Boesze-Battaglia K, Williams DS. The cell biology of the retinal pigment epithelium. Prog Retin Eye Res 2020
[Epub ahead of print]

13. Klimanskaya I, Hipp J, Rezai KA, West M, Atala A, Lanza R. Derivation and comparative assessment of retinal pigment epithelium from human embryonic stem cells using transcriptomics. Cloning Stem Cells 2004;6:217-245

14. Kashani AH. Stem cell therapy in nonneovascular age-related macular degeneration. Invest Ophthalmol Vis Sci 2016;57:ORSFm1-9

15. Nazari H, Zhang L, Zhu D, Chader GJ, Falabella P, Stefanini F, Rowland T, Clegg DO, Kashani AH, Hinton DR, Humayun MS. Stem cell based therapies for age-related macular degeneration: the promises and the challenges. Prog Retin Eye Res 2015;48:1-39

16. De Paiva MRB, Lage NA, Guerra MCA, Mol MPG, Ribeiro MCS, Fulgêncio GO, Gomes DA, Da Costa César I, Fialho SL, Silva-Cunha A. Toxicity and in vivo release profile of sirolimus from implants into the vitreous of rabbits' eyes. Doc Ophthalmol 2019;138:3-19

17. Grambergs R, Mondal K, Mandal N. Inflammatory ocular diseases and sphingolipid signaling. Adv Exp Med Biol 2019;1159:139-152

18. Hassan M, Karkhur S, Bae JH, Halim MS, Ormaechea MS, Onghanseng N, Nguyen NV, Afridi R, Sepah YJ, Do DV, Nguyen QD. New therapies in development for the management of non-infectious uveitis: a review. Clin Exp Ophthalmol 2019;47:396-417

19. Kaçmaz RO, Kempen JH, Newcomb C, Daniel E, Gangaputra S, Nussenblatt RB, Rosenbaum JT, Suhler EB, Thorne JE, Jabs DA, Levy-Clarke GA, Foster CS. Cyclosporine for ocular inflammatory diseases. Ophthalmology 2010;117: 576-584

20. Nguyen QD, Merrill PT, Sepah YJ, Ibrahim MA, Banker A, Leonardi A, Chernock M, Mudumba S, Do DV. Intravitreal sirolimus for the treatment of noninfectious uveitis: evolution through preclinical and clinical studies. Ophthalmology 2018;125:1984-1993

21. Lee YJ, Kim SW, Seo KY. Application for tacrolimus ointment in treating refractory inflammatory ocular surface diseases. Am J Ophthalmol 2013;155:804-813

22. Fang CB, Zhou DX, Zhan SX, He Y, Lin Z, Huang C, Li J. Amelioration of experimental autoimmune uveitis by leflunomide in Lewis rats. PLoS One 2013;8:e62071

23. Robertson SM, Lang LS. Leflunomide: inhibition of S-antigen induced autoimmune uveitis in Lewis rats. Agents Actions 1994;42:167-172

24. Ramos-Casals M, Brito-Zerón P, Bombardieri S, Bootsma H, De Vita S, Dörner T, Fisher BA, Gottenberg JE, Hernandez-Molina G, Kocher A, Kostov B, Kruize AA, Mandl T, Ng WF, Retamozo S, Seror R, Shoenfeld Y, SisóAlmirall A, Tzioufas AG, Vitali C, Bowman S, Mariette X. EULAR recommendations for the management of Sjögren's syndrome with topical and systemic therapies. Ann Rheum Dis 2020;79:3-18

25. Thomson JA, Itskovitz-Eldor J, Shapiro SS, Waknitz MA, Swiergiel JJ, Marshall VS, Jones JM. Embryonic stem cell lines derived from human blastocysts. Science 1998;282: 
$1145-1147$

26. Song MK, Lui GM. Propagation of fetal human RPE cells: preservation of original culture morphology after serial passage. J Cell Physiol 1990;143:196-203

27. Liu Y, Xu HW, Wang L, Li SY, Zhao CJ, Hao J, Li QY, Zhao TT, Wu W, Wang Y, Zhou Q, Qian C, Wang L, Yin ZQ. Human embryonic stem cell-derived retinal pigment epithelium transplants as a potential treatment for wet age-related macular degeneration. Cell Discov 2018;4:50

28. Schwartz SD, Hubschman JP, Heilwell G, Franco-Cardenas V, Pan CK, Ostrick RM, Mickunas E, Gay R, Klimanskaya I, Lanza R. Embryonic stem cell trials for macular degeneration: a preliminary report. Lancet 2012;379:713-720

29. Schwartz SD, Regillo CD, Lam BL, Eliott D, Rosenfeld PJ, Gregori NZ, Hubschman JP, Davis JL, Heilwell G, Spirn M, Maguire J, Gay R, Bateman J, Ostrick RM, Morris D, Vincent $M$, Anglade E, Del Priore LV, Lanza R. Human embryonic stem cell-derived retinal pigment epithelium in patients with age-related macular degeneration and Stargardt's macular dystrophy: follow-up of two open-label phase 1/2 studies. Lancet 2015;385:509-516

30. Sugino IK, Gullapalli VK, Sun Q, Wang J, Nunes CF, Cheewatrakoolpong N, Johnson AC, Degner BC, Hua J, Liu T, Chen W, Li H, Zarbin MA. Cell-deposited matrix improves retinal pigment epithelium survival on aged submacular human Bruch's membrane. Invest Ophthalmol Vis Sci 2011;52:1345-1358

31. Sugino IK, Sun Q, Wang J, Nunes CF, Cheewatrakoolpong N, Rapista A, Johnson AC, Malcuit C, Klimanskaya I, Lanza R, Zarbin MA. Comparison of FRPE and human embryonic stem cell-derived RPE behavior on aged human Bruch's membrane. Invest Ophthalmol Vis Sci 2011;52: 4979-4997

32. Mosmann T. Rapid colorimetric assay for cellular growth and survival: application to proliferation and cytotoxicity assays. J Immunol Methods 1983;65:55-63
33. Maminishkis A, Chen S, Jalickee S, Banzon T, Shi G, Wang FE, Ehalt T, Hammer JA, Miller SS. Confluent monolayers of cultured human fetal retinal pigment epithelium exhibit morphology and physiology of native tissue. Invest Ophthalmol Vis Sci 2006;47:3612-3624

34. Kokkinaki M, Sahibzada N, Golestaneh N. Human induced pluripotent stem-derived retinal pigment epithelium (RPE) cells exhibit ion transport, membrane potential, polarized vascular endothelial growth factor secretion, and gene expression pattern similar to native RPE. Stem Cells 2011;29: 825-835

35. Fronk AH, Vargis E. Methods for culturing retinal pigment epithelial cells: a review of current protocols and future recommendations. J Tissue Eng 2016;7:2041731416650838

36. Juuti-Uusitalo K, Vaajasaari H, Ryhänen T, Narkilahti S, Suuronen R, Mannermaa E, Kaarniranta K, Skottman H. Efflux protein expression in human stem cell-derived retinal pigment epithelial cells. PLoS One 2012;7:e30089

37. Luo Y, Zhuo Y, Fukuhara M, Rizzolo LJ. Effects of culture conditions on heterogeneity and the apical junctional complex of the ARPE-19 cell line. Invest Ophthalmol Vis Sci 2006;47:3644-3655

38. Zarbin MA. Analysis of retinal pigment epithelium integrin expression and adhesion to aged submacular human Bruch's membrane. Trans Am Ophthalmol Soc 2003;101: 499-520

39. Booij JC, Baas DC, Beisekeeva J, Gorgels TG, Bergen AA. The dynamic nature of Bruch's membrane. Prog Retin Eye Res 2010;29:1-18

40. Morizur L, Herardot E, Monville C, Ben M'Barek K. Human pluripotent stem cells: a toolbox to understand and treat retinal degeneration. Mol Cell Neurosci 2020;107:103523

41. Mannermaa E, Vellonen KS, Urtti A. Drug transport in corneal epithelium and blood-retina barrier: emerging role of transporters in ocular pharmacokinetics. Adv Drug Deliv Rev 2006;58:1136-1163 